\title{
Stochastic modeling and rate theory of atomic friction
}

\author{
Mykhaylo Evstigneev ${ }^{1}$, Juan J. Mazo ${ }^{2}$, and Peter Reimann ${ }^{1}$ \\ 1 Fakultät für Physik, Universität Bielefeld, 33615 Bielefeld, Germany; \\ 2 Departamento de Física de la Materia Condensada \\ and Instituto de Ciencia de Materiales de Aragón, \\ CSIC-Universidad de Zaragoza, 50009 Zaragoza, Spain
}

\begin{abstract}
Atomic friction involves objects whose dynamics is strongly influenced by thermal fluctuations. In stochastic modeling, one focuses on a few relevant degrees of freedom, whereas the atomistic ones are taken into account by introducing dissipation and noise. We review applications of this approach to atomic friction, namely, the basic Prandtl-Thomlinson model, some of its multidimensional generalizations, and the rate approximation, which allows one to obtain analytical results not easily accessible by other methods.
\end{abstract}

\section{INTRODUCTION}

Macroscopic friction between solids is well known to be both of paramount practical importance and of notorious difficulty regarding its theoretical understanding [1-4]. While macroscopic friction involves interactions between numerous asperities of the two contacting surfaces, employing an atomic force microscope (AFM) offers a unique opportunity to probe the frictional forces between a single asperity - the tip of an AFM cantilever - and an atomically flat surface. Therefore the research direction of friction force microscopy (FFM) [5] had been initiated only a year after the invention of the AFM in 1986 [6] and is being intensively pursued since then (see the reviews [7-13] and references therein).

In a typical FFM experiment [5], the tip of an AFM cantilever is brought in contact with an atomically clean surface by means of a normal load $F_{N}$, while the cantilever base is set in motion at a constant velocity $v$ (see Fig. 1). The interaction between the tip and the surface leads to a torsional deformation of the cantilever. One can determine the magnitude

${ }^{*}$ E-mail: mykhaylo@physik.uni-bielefeld.de 


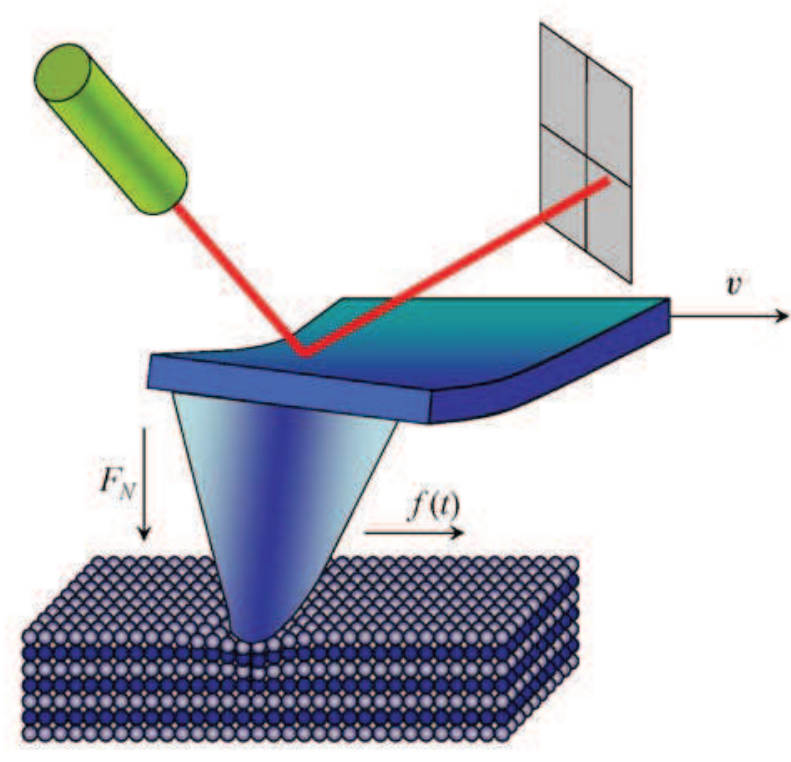

FIG. 1: Schematic illustration of an FFM experiment. Note that this picture is severely out of scale: in a real experiment, the tip radius is of the order of $10 \mathrm{~nm}$, and the contact region consists of several hundreds of atoms.

of this deformation by optical means and thus deduce the resulting elastic force $f(t)$, which, by Newton's third law, equals the instantaneous force of friction. The central quantity of interest is the time-averaged friction force

$$
\bar{f}:=\lim _{t \rightarrow \infty} \frac{1}{t} \int_{0}^{t} d t^{\prime} f\left(t^{\prime}\right)
$$

While it has been known from the time of Coulomb that the force of friction between two macroscopic bodies in contact is independent of their relative velocity, friction force on the nanoscale exhibits a non-trivial velocity dependence, which will be discussed in this contribution.

Experimental results reveal that the effects of thermal noise play an important role in nanoscale friction. Understanding such a phenomenon is the most important challenge of the stochastic modeling of atomic friction. The specific indications of the importance of thermal effects are thermal fluctuations of the instantaneous friction force $f(t)$ and, in particular, randomness of interstitial jumps of the cantilever in the so-called stick-slip regime of motion, the temperature dependence of atomic friction, and the approximately logarithmic dependence of the friction force, which can be explained using a model based on the assump- 
tion that the tip transitions (slips) from one lattice site to the next are due to thermally activated rate processes $[10,14-18]$.

Though simpler than macroscopic friction, the adequate interpretation and modeling of nanofriction experiments still represents a formidable challenge. In particular, direct molecular dynamics (MD) simulations, initiated in the mid-nineties [19, 20], have only recently started to approach experimentally realistic pulling velocities. Without resorting to special techniques, the velocities accessible in MD studies are in the $1-10^{3}$ meter per second range [21], orders of magnitude too fast in comparison with the experimentally relevant values. Smaller velocities can be probed with the help of methods that accelerate the algorithm performance, such as parallel replica dynamics [22, 23] (velocity of a few $\mathrm{mm} / \mathrm{s}$ ), or its combination with hyperdynamics $[24,25]$ that has further reduced the pulling velocities to the experimental microns per second. At the same time, the number of atoms that can be simultaneously accounted for in MD is still several orders of magnitude smaller than in the experiment. Last but not least, MD simulations may take up to several weeks of computational time. The reason for these limitations is the enormous time scale separation between atomic vibrations proceeding on the subpicosecond time scale, and sliding motion of the tip, which covers only a few lattice constants in a millisecond.

Hence, non-trivial theoretical modeling steps are indispensable, in particular the concepts of non-linear stochastic processes [26-31]; the above-mentioned time-scale separation justifies and greatly facilitates the calculations within such models. In stochastic modeling, one focuses on just a few relevant degrees of freedom, which, in the case of nanofriction, describe the tip geometric configuration. The huge number of the remaining atomistic degrees of freedom are accounted for by introducing the effects of randomness and dissipation in the tip equations of motion. In this contribution, we review two different types of stochastic approaches to nanofriction modeling, both stemming from the early works due to Prandtl [32] and Tomlinson [33]: one is based on the Langevin equation, and the other on the theory of thermally activated rate processes. 


\section{LANGEVIN MODELING}

\section{A. Langevin equation}

The system from Fig. 1, though small, still involves a huge number of microscopic degrees of freedom. In a one-dimensional model, the experimentally observable lateral force $f(t)$ can be deduced from the torsional deformation of the cantilever and is directly related to the displacement $x(t)-v t$ of the AFM tip from its equilibrium position $v t$ at a moment of time $t$, cf. Eq. (5) below. To obtain the evolution equation for this relevant collective degree of freedom $x(t)$, one writes down the equation of motion for all coordinates of the system, and then projects the system's microscopic state onto the subspace characterized by a given value of $x(t)$ [26]. As a result of this procedure, an equation of motion for $x(t)$ is obtained, in which the effect of atomistic degrees of freedom is accounted for by introduction of the following objects: (i) a free-energy type potential $U(x, t)$ of mean force, (ii) memorydependent dissipative force, and (iii) a random force (noise) of finite correlation time. In view of the fact that the characteristic frequency associated with the collective variable $x(t)$ is of the order of $10^{5} \mathrm{~Hz}$, i.e. many orders of magnitude lower than the Debye frequencies describing the time-scale of the atomistic degrees of freedom, both the memory effects in dissipative force and the finite noise correlation time can be neglected.

The potential of mean force $U(x, t)$ consists of two contributions, the first one accounting for the elastic deformations of AFM and substrate, and the second for the tip-substrate interaction. Since the elastic deformations are typically small [34], we may neglect anharmonic effects in the elastic energy. Furthermore, we can assume that interaction only depends on the relative tip-substrate position $x$. We thus arrive at the approximation

$$
U(x, t)=\frac{\kappa_{L}(x-v t)^{2}}{2}+U(x)
$$

The argument in the first term indicates that the cantilever moves at a constant velocity $v>0$ to the right (cf. Fig. 1). Furthermore, focusing on an ideally flat atomic surface with lattice constant $a$ in $x$-direction, we conclude that $U(x)$ is invariant under a displacement by one period,

$$
U(x+a)=U(x)
$$

The lateral spring constant $\kappa_{L}$ describes the combined effect of the elastic deformation of 
the cantilever, the tip and the elastically deformed surface in the contact region [34-39]:

$$
\frac{1}{\kappa_{L}}=\frac{1}{\kappa_{\text {cantilever }}}+\frac{1}{\kappa_{\text {tip }}}+\frac{1}{\kappa_{\text {surface }}} .
$$

The experimentally observable lateral force $f(t)$ can be identified, according to Newton's third law, with the negative of the force caused by the elastic deformations, i.e.

$$
f(t)=-\kappa_{L}(x(t)-v t)
$$

Next, let us consider the elastic deformations of the cantilever, and, in particular, those of the tip apex (see Fig. 1). If these deformations, or equivalently, the state variable $x(t)$, are changing adiabatically slowly, then the system is at every instance of time in a thermal equilibrium state, i.e., we are dealing with a reversible process. If these changes are taking place at a finite speed, but still slowly enough that the thermal bath of the cantilever's atoms always remains close to the instantaneous accompanying thermal equilibrium, the remaining "small amount of disequilibrium" renders the process "slightly irreversible" and hence gives rise to a linear-response type dissipative force which, in the frame of reference of the cantilever, will be proportional to the velocity of the tip relative to the cantilever base. In the laboratory frame, this dissipative force assumes the form

$$
F_{c}(t)=-\eta_{c}(\dot{x}(t)-v)
$$

with an effective coupling strength $\eta_{c}>0$ between the collective coordinate $x$ and the close to equilibrium "cantilever and tip bath" (subscript "c"). In particular, because of the smallness of the tip deformations [34], the implicitly assumed independence of $\eta_{c}$ on the state $x(t)$ of the system is well justified. In a similar manner, the influence of the microscopic degrees of freedom of the substrate will result in a dissipative force $F_{s}(t)$, which is proportional to the tip velocity with respect to the substrate with the proportionality coefficient $\eta_{s}$ :

$$
F_{s}(t)=-\eta_{s} \dot{x}(t)
$$

Finally, we come to the randomly fluctuating forces acting on the slow state variable $x(t)$. They have the same origin as the dissipative forces, namely, the large number of fast degrees of freedom of the cantilever, tip and substrate baths. Due to this common origin and the fact that the baths always remain close to thermal equilibrium, one can show that those randomly fluctuating forces are completely fixed (in the statistical sense) by the 
functional form of the dissipative forces via the fluctuation-dissipation theorem [31, 40, 41]. Namely, the thermal "cantilever-and-tip-noise" acts on $x(t)$ in the usual form [26-30] of a fluctuating force $\sqrt{2 \eta_{c} k_{B} T} \xi_{c}(t)$ with temperature $T$, Boltzmann constant $k_{B}$, and unbiased $\delta$ correlated Gaussian noise $\xi_{c}(t)$ of unit strength. Similarly, the substrate gives rise to thermal fluctuations of the form $\sqrt{2 \eta_{s} k_{B} T} \xi_{s}(t)$ with an unbiased $\delta$-correlated Gaussian noise $\xi_{s}(t)$ independent of $\xi_{c}(t)$ :

$$
\left\langle\xi_{c}(t) \xi_{c}\left(t^{\prime}\right)\right\rangle=\left\langle\xi_{s}(t) \xi_{s}\left(t^{\prime}\right)\right\rangle=\delta\left(t^{\prime}-t\right), \quad\left\langle\xi_{c}(t) \xi_{s}\left(t^{\prime}\right)\right\rangle=0 .
$$

Essentially, the uniqueness of these thermal noises follows from the fact that any deviation from the above specified statistical properties could be exploited to construct a perpetuum mobile of the second kind [41]. Their independence is an approximation which is well justified by the fact that the contact between the two baths consists of comparatively few atoms.

Collecting all acting forces, we arrive at the following equation of motion [42, 43]:

$$
m \ddot{x}(t)+\eta \dot{x}(t)=-U^{\prime}(x(t))-\kappa_{L}(x(t)-v t)+\eta_{c} v+\sqrt{2 \eta k_{B} T} \xi(t) .
$$

where $m$ is the relevant effective mass associated with inertia effects of cantilever, tip, and substrate, and

$$
\eta:=\eta_{s}+\eta_{c}
$$

is the total damping coefficient. The zero-noise limiting case of this equation of motion is essentially equivalent to the early model of friction due to Prandtl [32] and Tomlinson [33], whereas the thermal noise term was introduced $[15,16]$ about seventy years after Prandtl and Tomlinson's publications. Such an equation (or its noise-free version) has been considered in a number of papers, the important difference being that either $\eta_{c}$ or (much less often) $\eta_{s}$ was assumed to vanish, whereas, in general, there is no reason to expect that any of these coefficients is zero. One can, however, straightforwardly relate the results obtained for arbitrary $\eta_{s}, \eta_{c}$ to those where either of them is set to zero. For instance, one can introduce a time translation $\tilde{t}=t+\eta_{c} / \kappa_{L}$ in (9), which eliminates the $\eta_{c} v$ term from the equation and allows one to express $\bar{f}\left(\eta_{s}, \eta_{c}\right)=\bar{f}\left(\eta_{s}+\eta_{c}, 0\right)-\eta_{c} v$.

It is instructive to rewrite Eq. (9) in the co-moving reference frame defined by

$$
z(t)=x(t)-v t, \quad \dot{z}(t)=\dot{x}(t)-v,
$$

in which the equation of motion assumes the form

$$
m \ddot{z}(t)+\eta \dot{z}(t)=-U^{\prime}(z(t)+v t)-\kappa_{L} z(t)-\eta_{s} v+\sqrt{2 \eta k_{B} T} \xi(t)
$$


and the instantaneous friction force from (5) becomes

$$
f(t)=-\kappa_{L} z(t)
$$

Then, the term $\eta_{s} v$ can be eliminated by a change of variables $\tilde{z}=z-\eta_{s} v / \kappa_{L}, \tilde{t}=t+\eta_{s} / \kappa_{L}$, so that $\bar{f}\left(\eta_{s}, \eta_{c}\right)=\bar{f}\left(0, \eta_{s}+\eta_{c}\right)+\eta_{s} v$. One of the consequences of Eq. (12) is that at high velocities the third term in the right-hand side, which describes the viscous drag of the substrate, exceeds all the other acting forces, and the force of friction (13) behaves as

$$
\bar{f} \rightarrow \eta_{s} v \text { for } v \rightarrow \infty
$$

allowing us, at least in principle, to experimentally determine the coefficient $\eta_{s}$ associated with the substrate from the slope of the force-velocity plot at high $v$.

\section{B. Parameter values}

In the simplest version, the potential $U(x)$ is taken to be sinusoidal,

$$
U(x)=-\frac{\Delta U}{2} \cos \frac{2 \pi x}{a}
$$

with a lattice constant $a \cong 0.2 \ldots 0.5 \mathrm{~nm}$ and a corrugation depth $\Delta U$, which varies from values close to zero to ca. $0.1 \mathrm{nN} \cdot \mathrm{nm}$, depending on the conditions of the experiment one wishes to model [44]. Note that, although a single-harmonic potential (15) is the one that is used most often in modeling, other possibilities have also been considered in the literature, e.g. a potential with sharp minima and flat maxima [45], quasiperiodic potentials [46, 47], potentials with localized Gaussian perturbations [48], fractal potentials [49], etc.

The value of the effective stiffness, $\kappa_{L}$, can be experimentally established from the slope of the force-distance curve in the regime where the cantilever is "stuck" to some lattice site of the substrate while its base moves at a constant velocity; then, the force evolves according to $f(t) \cong \kappa_{L} t$, up to an additive constant and small fluctuations [see Eqs. (17), (18) below for a more precise estimate]. This procedure yields, for various experimental conditions, the value of $\kappa_{L}$ between $0.1 \mathrm{~N} / \mathrm{m}$ and $10 \mathrm{~N} / \mathrm{m}[14,17,50]$.

With respect to the mass parameter, $m$, in the Langevin equation (9), its naive identification with the total mass of the cantilever is unjustified, which already becomes obvious from the fact that the effective stiffness $\kappa_{L}$ is at least one order of magnitude smaller than 
the "bare" torsional stiffness of the cantilever. This fact suggests that only a very small portion of the tip at the apex experiences a significant deformation, while most of the cantilever is relatively rigid during its motion. The mass parameter usually employed in the simulations is of the order of $m \cong 10^{-12} \mathrm{~kg}$ : this estimate follows from the fundamental torsional resonance frequency of the cantilever in contact with the sample, $(2 \pi)^{-1} \sqrt{\kappa_{L} / m}$, which has a typical value of ca. $400 \mathrm{kHz}$ [51]. It has also been proposed [42, 43] that in many experiments, the relevant mass parameter is so small that an overdamped limit $(m \rightarrow 0)$ is an adequate approximation.

Finally, the damping coefficient $\eta$ is difficult to measure directly, because the damping force $-\eta v$ is typically very small. Various estimates $[15,42,43,52,53]$, however, agree within an order of magnitude and yield $\eta \cong 10^{-6} \ldots 10^{-5} \mathrm{~kg} / \mathrm{s}$. Note that this value is close to the critical damping of the cantilever, $2 \sqrt{\kappa_{L} m}$; thus, depending on the experimental conditions, the motion of the cantilever may be either slightly underdamped or overdamped.

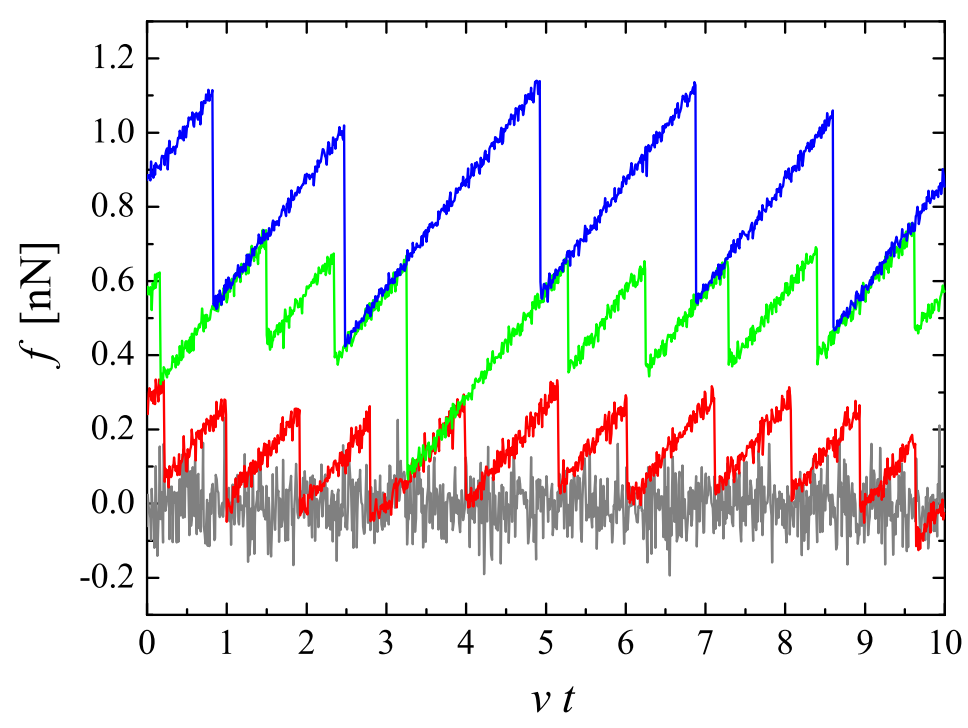

FIG. 2: Evolution of the friction force obtained from numerical simulation of the Langevin equation (9) with the following parameters: $a=0.3 \mathrm{~nm}, \kappa_{L}=1 \mathrm{~N} / \mathrm{m}, m=10^{-12} \mathrm{~kg}, \eta_{s}=\eta_{c}=10^{-6} \mathrm{~kg} / \mathrm{s}$, $v=0.1 \mu \mathrm{m} / \mathrm{s}, T=300 \mathrm{~K}$. The curves are obtained for different values of the potential corrugation depth, $\Delta U=2,50,100$, and $150 \mathrm{pN} \cdot \mathrm{nm}$ (from bottom to top) and feature smooth sliding (gray), stick-single slip motion (red), a mixture of single and double slip events (green), and stick-double slip motion (blue). 


\section{Regimes of motion}

Depending on the relative importance of cantilever mass, damping, spring constant, pulling velocity, potential corrugation amplitude, and temperature, different dynamical regimes can be achieved: continuous sliding [44], thermolubricity [54], regular stick-slip motion [15, 16], stick-multislip [52, 55-57], chaotic motion [58, 59], etc. Here, we will briefly describe the regimes of continuous sliding and stick-(multi)slip, as they have attracted most of the experimental interest so far.

Whether the cantilever will slide or perform stick-slip motion depends on the ratio of the maximal potential curvature, $\max _{x}\left|U_{0}^{\prime \prime}(x)\right|$, to the stiffness $\kappa_{L}$, also known as the PrandtlTomlinson parameter $[44,56]$. For a sinusoidal potential (15), it is given by

$$
\gamma_{P T}=\frac{2 \pi^{2} \Delta U}{a^{2} \kappa_{L}}
$$

For $\gamma_{P T}<1$, the potential (2) has a single minimum located roughly at $v t$, implying smooth sliding of the tip. In this regime, the force fluctuates around the value $\eta_{s} v$, see Fig. 2 , gray curve. On the other hand, if $1<\gamma_{P T}<4.604 \ldots$, the potential (2) becomes bistable. Further increase of $\gamma_{P T}$ to a value between ca. 4.604 and $7.788 \ldots$ results in a potential (2) having three minima; for $7.788<\gamma_{P T}<10.95 \ldots$, the potential has four minima, etc. [56].

The multistability of the potential (2) implies the possibility of stick-slip motion, whose physical picture is as follows. In a stick phase, the tip apex is confined to the $n$th lattice site, while the cantilever base moves at a velocity $v$, leading to an approximately linear (up to thermal and instrumental noise) increase of the elastic force and a reduction of the energy barrier separating the tip from the next lattice site. At some point, thermal noise drives the tip over that energy barrier into the next, $(n+1)$ st lattice site in a single slip event (see Fig. 2, red curve), whereas the kinetic energy of the tip is dissipated into the atomistic degrees of freedom and the force drops by a fixed amount. Then, a new stick phase begins. If inertia of the tip is large, then the tip may not be able to dissipate all of its kinetic energy in a single slip event and, as a result, the tip will perform a jump over two or even more lattice constants [38]. In general, the multiplicity of slips for a given value of $\gamma_{P T}$ is smaller than the number of minima of the total potential (2). The force evolution in the stick-multislip regime is exemplified in Fig. 2, showing a mixture of single and double slips (green curve) and pure double slips (blue curve). 
In the stick phases, the force increases according to

$$
f_{n}(t)=\kappa(v t-n a)
$$

with the rate of force increase characterized by a renormalized stiffness $\kappa$ given by [44, 60]

$$
\frac{1}{\kappa}=\frac{1}{\kappa_{L}}+\frac{1}{U^{\prime \prime}(b)}
$$

The parameter $b$ can be taken as the position of the minimum of the corrugation potential (15), e.g., $b=0$ [44]. A better accuracy is achieved if one identifies it with the position of the minimum of the total potential (2) corresponding to the mean force value $\bar{f}$ from Eq. (1), i.e.

finds $b$ from the relation $U^{\prime}(b)=\bar{f}$ [50]. Calculations show that the renormalized stiffness $\kappa$ weakly depends on velocity $v$ and may be smaller than the "bare" counterpart $\kappa_{L}$ by at most $10 \%[50]$.

In the stick-slip regime, the friction force decreases with temperature due to a reduction of the cantilever force at the onset of the slip event. However, in general, the situation can be more complex and in some cases a temperature-induced enhancement of nanoscale friction was predicted $[46,48,61]$ due to the effect of temperature on the slip length. Friction reduction is more significant at low velocities and moderate damping values. A change in the slip length is relevant for small enough spring constants (large $\gamma_{P T}$ ), where the total potential profile shows multiple accessible metastable states [57].

Multiple slip events have been observed experimentally $[5,52,56]$; it has also been suggested that the statistics of multislip events can be used to estimate the damping coefficient $\eta$ in the Langevin equation [52]. On the other hand, the majority of experimental studies that we are aware of focus on the stick-single slip regime of motion; moreover, some publications [44, 62] state explicitly that only single-slip, but no multiple slip events were detected in the measurements. This suggests that, in those studies, the tip dynamics is overdamped or the effective potential (2) is bistable.

\section{Some generalizations of the standard PT model}

\section{Disordered potential}

One of the biggest advantages of the PT model is its flexibility. With suitable modifications, it can be applied to study many variants of the nanofriction set-up. For instance, the 
standard PT model is characterized by a sinusoidal tip-substrate interaction potential (15) and corresponds to a perfectly periodic substrate lattice. However, other cases are worth to be analyzed, such as quasiperiodic lattices [46, 47] and lattices including defects [48, 61]. The presence of disorder or defects changes the local potential profile by modifying the potential barriers to overcome by the tip in every stick-slip cycle, and, on the other hand, it can also change the length of different slip events. Depending on parameter values, both effects cooperate or compete to change the friction force. This is especially noticeable at low temperatures, while thermal fluctuations at high enough temperatures (close to room temperature) can screen other effects.

\section{Additional slow degrees of freedom}

As discussed in Section II B, the cantilever in contact with the substrate is extremely soft in the apex region. Correspondingly, it is a natural extension of the PT model to consider the apex and the tip as two separate objects connected by a spring. This consideration leads to the so-called two-mass-two-spring models, which have been introduced and analyzed recently [63-67]. A multitude of friction regimes have been discovered within such a two-dimensional extension of the PT model, see Ref. [64] for a comprehensive review.

The assumption implicit in the Langevin equation (9) is that, during a nanofriction experiment, the tip moves along a one-dimensional manifold, whereas the substrate is, obviously, a two-dimensional object. Therefore, friction effects have been studied in two-dimensional geometries, with the main issue being friction force as a function of the tip-motion angle $[52,57,68-71]$. The comparison of experimental and theoretical models at finite temperatures is an active current research topic.

\section{AC actuation effects}

Ultrasonic vibrations have been proposed as a valid method for reducing friction at the nanoscale [72-77]. The PT model can be useful to study the dynamics of the system in the presence of in-plane and out-of-plane actuation fields. In the context of the model, in the first case the support position is affected by a shaking term. For out-of-plane actuation, the tip-substrate potential amplitude is modulated by an oscillating term [76], or the tip- 
sample distance is taken explicitly into account with a corresponding modification of the tip potential [72]. As in the regular case, thermal fluctuations are incorporated in the model as an additive Gaussian noise.

The most important finding is the existence of a wide medium-frequency range $(\sim \mathrm{kHz})$, where friction force is significantly reduced and even almost suppressed for intense enough actuation. The lower bound of this friction-reduced zone is determined by the inverse time to cover one lattice constant, $v / a$, and the upper bound by the effective damping.

\section{E. Friction force - velocity relations}

\section{Stratonovich formula}

In the asymptotic case of very low effective stiffness $\kappa_{L}$, the magnitude of force fluctuations, which is of the order of $\kappa_{L} a$, is also small, so that one can approximately replace the instantaneous elastic force (5) in Eq. (9) with its average value, $\bar{f}$. Furthermore, if one considers the overdamped $(m \rightarrow 0)$ limit $[42,43]$, then the Langevin equation (9) assumes the form

$$
\eta \dot{x}(t)=-U^{\prime}(x)+\bar{f}+\eta_{c} v+\sqrt{2 \eta k_{B} T} \xi(t)
$$

that describes the diffusion of a Brownian particle in a tilted periodic potential $U_{\text {tilted }}(x)=$ $U(x)-\left(\bar{f}+\eta_{c} v\right) x$. The problem of finding the average velocity of such a particle, $\langle\dot{x}\rangle \equiv v$, has been solved by Stratonovich, who derived the analytic formula [78]

$$
v=\frac{a k_{B} T\left(1-e^{-a\left(\bar{f}+\eta_{c} v\right) / k_{B} T}\right)}{\eta \int_{0}^{a} d x_{1} \int_{x_{1}}^{x_{1}+a} d x_{2} e^{\left[U\left(x_{1}\right)-U\left(x_{2}\right)+\left(x_{1}-x_{2}\right)\left(\bar{f}+\eta_{c} v\right)\right] / k_{B} T}} .
$$

The argument of the function in the right-hand side is not the average force $\bar{f}$, but rather the combination $\bar{f}+\eta_{c} v$. In order to plot the $\bar{f}-v$ relation, one can, first, for each given value of the combined force $\bar{f}+\eta_{c} v$ calculate the corresponding velocity $v$ using the Stratonovich formula (20), and then deduce the average friction force $\bar{f}$ corresponding to this velocity value by subtracting the value of $\eta_{c} v$ from the combined force. Apart from the result (20), we are not aware of any exact force-velocity relation applicable to the general case of arbitrary inertia $m$ or effective stiffness $\kappa_{L}$. 


\section{General case}

In a number of works, the Langevin equation (9) was simulated numerically using a random number generator. In the stick-slip regime, the ensuing relation between the friction force (1) and pulling velocity was found to be logarithmic at fast pulling, where the cantilever performs only forward slips, namely,

$$
\bar{f} \propto\left|\ln \left(v / v_{0}\right)\right|^{\alpha}
$$

where $v_{0}$ is some reference velocity. Fitting the simulation results with an expression of the type (15) yielded the exponents $\alpha$ close to unity [68]. From a theoretical perspective, the exponent $\alpha$ is related to the functional form for a potential barrier decrease as the cantilever base moves. The $\alpha=1$ value [14] is achieved for a barrier decreasing linearly with the force (Bell-type expression [79]), whereas the exponent $\alpha=2 / 3$ results from a linear-cubic approximation of the potential at small barriers $[15,16]$. It has been recently pointed out $[80]$ that the regimes corresponding to these two values of $\alpha$ can be distinguished only if one can probe a wide range of velocities covering many decades, whereas the typical experimentally accessible velocity range (usually not more than three decades) is too narrow to determine $\alpha$ unambiguously.

In the slow-pulling regime, the back-slips of the cantilever play a significant role, and the linear-response arguments predict that

$$
\bar{f} \propto v
$$

with the proportionality constant being different from the substrate damping coefficient $\eta_{s}$ (unless the potential corrugation is zero). Recently, it has been suggested [80] that the two regimes, logarithmic (21) and linear (22), can be unified by a phenomenological ansatz of the type

$$
\bar{f}=f_{0}(T) \sinh ^{-1}\left[v / v_{0}(T)\right]
$$

with $f_{0}(T) \propto T^{2 / 3}$ and $v_{0}(T) \propto T$. The accuracy of this ansatz has been demonstrated for a velocity range covering about seven decades [80].

The logarithmic (or $\sinh ^{-1}$ ) force-velocity relation can be obtained within a general framework of the rate theory, which is the subject of the next section. 


\section{RATE THEORY}

\section{A. Rate equation}

The Langevin equation (9) can be obtained, at least formally, by projecting the microscopic state of the system onto a subspace characterized by given values of the slow collective degrees of freedom. If the total state space consists of many "regions of attraction", such that the system spends most of the time within any such region and only rarely performs transitions between them, then an even more coarse-grained description is possible, namely, the one that uses the language of occupation probabilities of such regions and transition rates between them [29]. This is the case for the cantilever stick-slip motion: the average time spent by the cantilever within a given lattice site, $a / v$, is of the order of $0.1 \mathrm{~ms}$, whereas the timescale of the tip coordinate fluctuations, $x(t)$, can be estimated as the inverse reso-

nance frequency, $\sqrt{m / \kappa_{L}}$, or, in the overdamped case, as the tip relaxation time, $\eta / \kappa$, and is at least two orders of magnitude faster for both estimates.

In view of Fig. 2, the elastic force (5) in the $n$th stick phase of the stick-slip motion can be naturally separated into two contributions, regular and random:

$$
f(t)=f_{n}(t)+\delta f(t)
$$

where the regular part, $f_{n}(t)$, is the force (17) corresponding to a minimum of the combined potential (2), and the random part $\delta f(t)$ results from the tip fluctuations about that minimum. The rate of interstitial slips, i.e. the slip probability per unit time, depends on the regular part of the force, $f_{n}(t)$, whereas the fluctuating part, $\delta f(t)$, becomes irrelevant in this coarse-grained picture. We will denote the forward rate out of the $n$th lattice site as $\omega_{+}\left(f_{n}\right)$. If the elastic force is not too high, the cantilever can also perform a back-transition into the previous lattice site with the backward rate $\omega_{-}\left(f_{n}\right)$. For symmetric substrates, the two rates, $\omega_{+}\left(f_{n}\right)$ and $\omega_{-}\left(f_{n}\right)$, are related: the rate to jump forward "along the force" equals the rate to jump backward "against the force", i.e. $\omega_{+}\left(f_{n}\right)=\omega_{-}\left(-f_{n}\right)$. Furthermore, both rates are given by the Kramers-Arrhenius law [29]

$$
\omega_{+}\left(f_{n}\right)=\omega_{-}\left(-f_{n}\right)=\omega_{0} e^{-\Delta U\left(f_{n}\right) / k_{B} T},
$$

where $\Delta U\left(f_{n}\right)$ is the force-dependent height of the energy barrier separating the current minimum from the next one, and the prefactor $\omega_{0}$ depends usually quite weakly on force 
and temperature.

Neglecting the possibility of multiple slips, the probability $p_{n}(t)$ for the tip to find itself in the $n$th lattice site at the moment of time $t$ obeys the rate (or master) equation [81, 82]:

$$
\dot{p}_{n}(t)=-\left[\omega_{+}\left(f_{n}(t)\right)+\omega_{-}\left(f_{n}(t)\right)\right] p_{n}(t)+\omega_{+}\left(f_{n-1}(t)\right) p_{n-1}(t)+\omega_{-}\left(f_{n+1}(t)\right) p_{n+1}(t) .
$$

Here, the first term in the right-hand side describes the transitions out of the $n$th lattice site into the next $(n+1)$ st and the previous $(n-1)$ st ones, and the remaining two terms correspond to transitions into the $n$th lattice site from the $(n-1)$ st and the $(n+1)$ st ones.

We are interested in the long-time limiting solution of the rate equation (1), which is established after the decay of transient processes. In this limit, the probability to find the tip in the $n$th lattice site at the moment of time $t$ is the same as the probability to find it in the previous lattice site at the earlier time, shifted by an interval $a / v$ necessary for the cantilever base to cover one lattice constant $a$ at the velocity $v$ :

$$
p_{n}(t)=p_{n-1}(t-a / v)
$$

We define the force probability distribution, $p(f)$, as the probability to find the tip in the $n$th lattice site at that moment of time $t$ when the corresponding elastic force $f_{n}(t)$ has the given value $f$, i.e. $f_{n}(t)=f$. According to Eq. (17), this time is given by $t=f /(\kappa v)+n a / v$, so that

$$
p(f)=p_{n}(f /(\kappa v)+n a / v), \quad p_{n}(t)=p(f-n \kappa a) .
$$

In view of the relation $(27)$, the so defined $p(f)$ is independent of the index $n$. It can be shown [82] that the time-averaged value of any function of force, $g(f)$, can be expressed as

$$
\langle g(f)\rangle=\frac{1}{\kappa a} \int_{-\infty}^{\infty} d f g(f) p(f),
$$

i.e. the function $p(f) /(\kappa a)$ has the physical meaning of force probability density.

The normalization condition expresses the fact that the tip finds itself in some lattice site with probability one for all times:

$$
\sum_{n=-\infty}^{\infty} p_{n}(t)=\sum_{n=-\infty}^{\infty} p(f+n \kappa a)=1
$$

In the second part, we used the second relation (28) and replaced $n \rightarrow-n$. 
Using the definition (28) and making the change of variables (17) in the rate equation (26), we find that the force probability distribution satisfies the rate equation in the force domain [82]:

$$
\kappa v p^{\prime}(f)=-\left[\omega_{+}(f)+\omega_{-}(f)\right] p(f)+\omega_{+}(f+\kappa a) p(f+\kappa a)+\omega_{-}(f-\kappa a) p(f-\kappa a) .
$$

\section{B. Validity conditions}

The rate approximation is valid if the system possesses two very different time scales: the fast one describing the relaxation of the tip within a given potential well and the much slower one describing the thermally activated interwell transitions of the tip. This is realized if the typical height of the barrier separating two adjacent potential minima is at least a few times larger than the thermal energy [29]:

$$
\Delta U(f) \gg k_{B} T
$$

This condition implies that the Prandtl-Tomlinson parameter (16) must satisfy $\gamma_{P T} \gg 1$. Furthermore, pulling must proceed sufficiently slowly to allow the slips to occur before the condition (32) is violated, which happens at some force $f_{\max }$ for which $\Delta U\left(f_{\max }\right)$ equals a few $k_{B} T$. According to an estimate from Ref. [83], the pulling velocity must therefore satisfy the condition

$$
v \ll-\frac{k_{B} T \omega\left(f_{\max }\right)}{\kappa \Delta U^{\prime}\left(f_{\max }\right)} .
$$

Pulling velocities $v$ bigger than in (33) lead to the onset of the opposite regime of steady sliding $[42,43]$ characterized by friction forces increasing as $\eta_{s} v$.

\section{Parameterization}

The parameter values used in the rate approach (26) can be derived from the Langevin equation (9) in the limit of deep corrugation $\Delta U$ or low $\kappa_{L}$. In particular, the effective stiffnesses in the two approaches, $\kappa$ and $\kappa_{L}$, are slightly different, see Eq. (18). The barrier height in the rate expression (25) can be approximated as

$$
\Delta U(f) \cong \Delta U_{0}\left(1-f / f_{c}\right)^{\beta}
$$


where $\Delta U_{0}$ is the barrier height at zero force, which is related to the corrugation depth $\Delta U$ of the potential (15), $f_{c}$ is the critical force value at which the barrier vanishes, and the exponent $\beta$ describing the barrier reduction with force depends on the functional form of the potential $U(x)$ from Eq. (2). Considering the sinusoidal potential (15), it is often assumed that the exponent $\beta=3 / 2$, which results from the linear-cubic interpolation of the potential $U(x)$ at forces $f$ slightly below $f_{c}[15,16]$. Note that, in practice, other $\beta$-values of the order of unity have also been either assumed to fit experimental or simulation results or deduced from such a fitting $[14,50,68,84]$.

For a sinusoidal potential (15), the critical force is given by [15-17]

$$
f_{c}=\pi \Delta U / a
$$

and usually does not exceed several nanonewtons. The height $\Delta U_{0}$ of the force-dependent potential barrier (34) at zero force was initially taken to be equal the corrugation depth $\Delta U$ of the coordinate-dependent potential energy (15) [15-17]. Such an approximation is valid, strictly speaking, in the limit of vanishingly small lateral stiffness $\kappa_{L}$, which is inherent in the Stratonovich formula (20). For a finite spring constant $\kappa_{L}$, an improved approximation is due to [85], namely

$$
\Delta U_{0}=\Delta U+\kappa_{L} a^{2} / 8
$$

still leaving $\Delta U_{0}$ and $\Delta U$ of comparable order of magnitude. We note that, while the expression (34) is a useful simple ansatz for the barrier height, other approximations also have been introduced, that lead to an almost perfect agreement of the results for the friction force obtained within the rate and Langevin approaches, see Refs. [60, 86] for details.

The expression for the rate prefactor $\omega_{0}$ depends on whether the tip dynamics is underdamped or overdamped [29]. In the practically important overdamped limit, it is [29]

$$
\omega_{0}=\frac{\sqrt{U_{m i n}^{\prime \prime}\left|U_{m a x}^{\prime \prime}\right|}}{2 \pi \eta},
$$

where $U_{\min , \max }^{\prime \prime}$ denote the curvature at the minimum and maximum of the potential (2) when the force (5) has the value $f$. More generally, the rate parameters can be derived from any multidimensional version of the Langevin equation (e.g. the two-mass-two-spring model [66]), and even from the all-atom description used in MD, where the heat-bath degrees of freedom are not "integrated out". This is achieved using the transition state theory, see [23] 
for details. While the energy parameter $\Delta U_{0}$ and the force parameter $f_{c}$ are more or less consistent in different publications, the parameter $\omega_{0}$ is found to vary in a wide range of values, from tens of $\mathrm{kHz}[17,50]$ to hundreds of $\mathrm{MHz}[62]$ and $\mathrm{GHz}[66]$.

\section{Types of the stick-slip motion}

Following Ref. [82], we describe here four regimes of the stick-slip motion that are inherent within the rate approach; see Fig. 3 for a numerical illustration obtained by simulating the stick-slip process using a Monte Carlo technique [82].

Perhaps, the most widely investigated type is realized for relatively high effective stiffness and relatively fast pulling, so that the back-transitions are negligible, see Fig. 3(a). The characteristic feature of such curves is the existence of a force interval, where the stick probability is close to 1 . In this regime, the dependence of the mean friction force on the pulling velocity is approximately logarithmic (21), reflecting the exponential dependence of the transition rate $\omega_{+}(f)$.

If pulling is slow, Fig. 3(b), the back-transitions become important and have indeed been observed experimentally [87]. For ultraslow pulling, they occur at almost the same frequency as the forward transitions, resulting in a linear force-velocity relation (22).

The velocity value at which the transition between the linear and logarithmic regimes occurs can be estimated from the following reasoning [82]. A typical stick phase begins at a force close to $\bar{f}-\kappa a / 2$. Therefore, the typical frequency of the back transitions is $\omega_{-}(\bar{f}-\kappa a / 2)=\omega_{+}(\kappa a / 2-\bar{f})$. Assuming that we are in the linear response regime where the mean force $\bar{f} \propto v$ is small, this can be approximated as $\omega_{+}(\kappa a / 2)$. The back-slips will not be observed if the time to cover one lattice constant is faster than the inverse of the back-slip frequency. Thus, the force-velocity relation will be approximately logarithmic with back-jumps playing practically no role for $v \gg a \omega_{+}(\kappa a / 2)$ and approximately linear for $v \ll a \omega_{+}(\kappa a / 2)$.

The two regimes exemplified by the stick-slip curves from Fig. 3(a) and (b) are realized if the forward rate is very small below some velocity-dependent force value and high above this force value. Then, once formed, a given stick phase will survive in a rather wide force interval where the rate is small. Once the force increases beyond this interval, the rate will become very large, and the tip will slip into the next lattice site, resulting in the force drop 

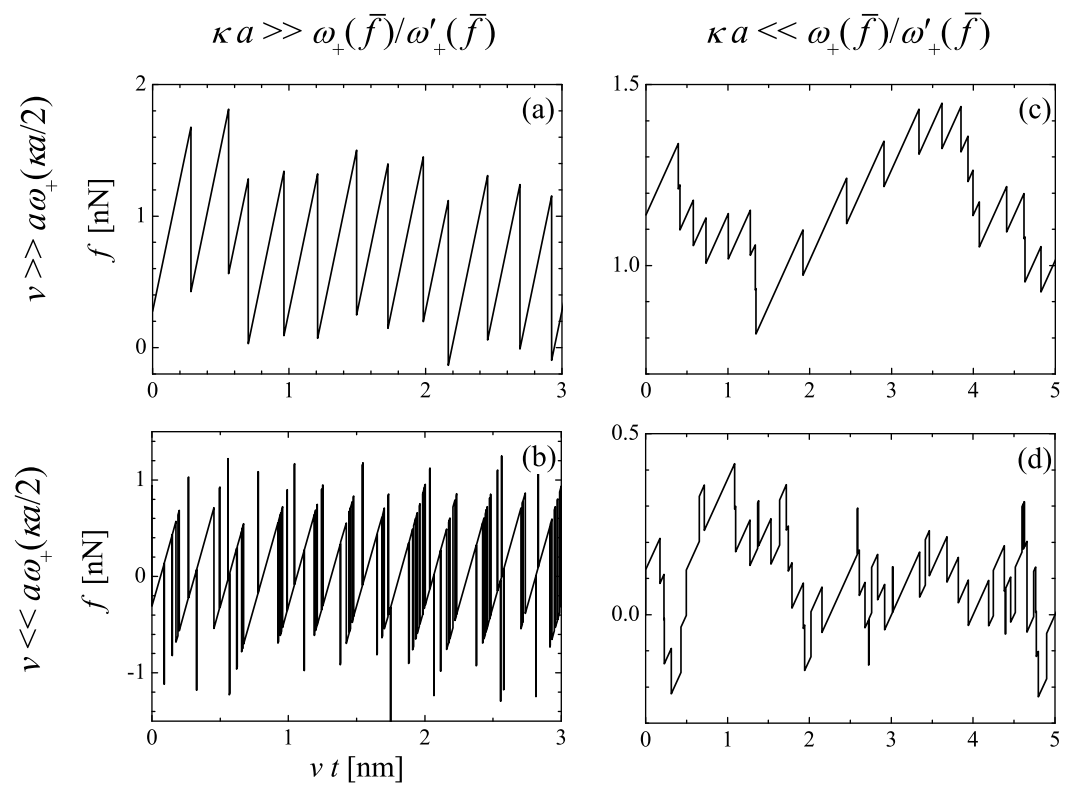

FIG. 3: The four basic types of stick-slip motion obtained by a numerical Monte Carlo simulation of the stick-slip process [82]. The rate parameters are as follows: $\omega_{0}=1 \mathrm{MHz}, \Delta U_{0}=10 k_{B} T$, $f_{c}=3 \mathrm{nN}, \beta=3 / 2, a=0.25 \mathrm{~nm}$. The effective stiffness $\kappa=5 \mathrm{~N} / \mathrm{m}$ for curves (a) and (b), and $\kappa=0.5 \mathrm{~N} / \mathrm{m}$ for (c) and (d). The pulling velocity $v=2 \mu \mathrm{m} / \mathrm{s}$ for curves (a) and (c), and $v=0.01$ $\mu \mathrm{m} / \mathrm{s}$ for curves (b) and (d).

by $\kappa a$, a strong rate reduction, and a small probability of the next slip event until the force $f$ reaches again the upper limit of that interval. Thus, the regimes shown in Fig. 3(a), (b) are realized if the rate varies strongly within the force interval of the order of $\kappa a$ around the mean force value $\bar{f}$, i.e. $\omega_{+}^{\prime}(\bar{f}) \kappa a \gg \omega_{+}(\bar{f})$.

In the opposite case, $\kappa a \ll \omega_{+}(\bar{f}) / \omega_{+}^{\prime}(\bar{f})$, the rate $\omega_{ \pm}(f)$ depends weakly on the applied force $f$. Then, right after some slip event, the probability to perform another slip remains significant, so that the short stick phases will be scattered in a rather broad force interval in such a manner that there is no particular force value that would be common to all of them. This situation is illustrated in Fig. 3(c) (fast pulling, no back-slips) and (d) (slow pulling, back-slips present). 


\section{E. Force-velocity relations}

\section{Most probable and average slip force at high $\kappa$}

In the regime of fast pulling and large stiffness exemplified by Fig. 3(a), all stick phases cross some common force interval. Any force value $f_{L}$ from that interval is characterized by the occupation probability $p\left(f_{L}\right)$ very close to one, and thus can be regarded as a "starting point" common to all stick phases. Then, a description even simpler than the rate equation (31) is possible $[14-17,50,83,88]$. Namely, one can ask about the probability $\mathcal{P}\left(t \mid t_{L}\right)$ of staying within the same lattice site up to the moment of time $t$, provided that the tip entered this site at the initial time $t_{L}$, i.e. $\mathcal{P}\left(t_{L} \mid t_{L}\right)=1$. The time evolution of $\mathcal{P}\left(t \mid t_{L}\right)$ for $t \geq t_{L}$ is governed by the rate equation, initially considered within the context of nanofriction in [14]

$$
\frac{\partial \mathcal{P}\left(t \mid t_{L}\right)}{\partial t}=-\omega_{+}(f(t)) \mathcal{P}\left(t \mid t_{L}\right)
$$

With the help of the transformation of variables (17), we find from the rate equation (38) the probability that the transition into the next site occurs at a force value between $f$ and $f+d f$, provided that the initial lower force value for a given stick phase was $f_{L}$,

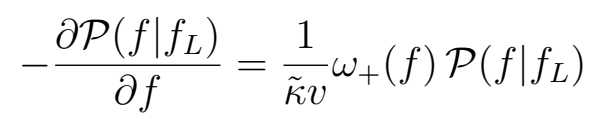

with the initial condition $\mathcal{P}\left(f_{L} \mid f_{L}\right)=1$. The most probable force $f_{*}$, at which the transition into the next site occurs, is evaluated by setting the second derivative of $\mathcal{P}\left(f \mid f_{L}\right)$ to zero. This results in the relation between the pulling velocity and the most probable force at the moment of slip:

$$
v=\frac{\omega_{+}^{2}\left(f_{*}\right)}{\kappa \omega_{+}^{\prime}\left(f_{*}\right)} .
$$

This equation in various forms has been presented in Refs. [14-17, 66], the difference between these works stemming from different assumptions regarding the functional dependence of $\omega_{+}(f)$ on $f$. For the rate ansatz (25) with a constant prefactor $\omega_{0}$ and the barrier height (34), the ensuing relation between the most probable slip force and the velocity is

$$
\ln \frac{v}{v_{0}}=-\frac{\Delta U_{0}}{k_{B} T}\left(1-\frac{f_{*}}{f_{c}}\right)^{\beta}-(\beta-1) \ln \left(1-\frac{f_{*}}{f_{c}}\right), \quad v_{0}=\frac{\omega_{0} f_{c} k_{B} T}{\beta \kappa \Delta U_{0}} .
$$

It can be solved analytically [83]:

$$
\frac{f_{*}}{f_{c}}=1-\left(\frac{\beta-1}{\beta} \frac{k_{B} T}{\Delta U_{0}} W(z)\right)^{1 / \beta}, z=\frac{\beta}{\beta-1}\left(\frac{\omega_{0} f_{c}}{\beta \kappa v}\right)^{\beta /(\beta-1)}\left(\frac{k_{B} T}{\Delta U_{0}}\right)^{1 /(\beta-1)}
$$


where $W(z)$ is Lambert function defined implicitly by

$$
W(z) e^{W(z)}=z
$$

Other approximations that have been introduced previously can be derived from the result (42). In particular, at large arguments $z$, the Lambert function behaves as a natural logarithm, thus leading to the asymptotic law (21) complemented by a simple relation between the exponents, $\alpha$ and $\beta$ :

$$
\alpha=1 / \beta
$$

With respect to the mean force $\bar{f}$, an accurate analytical approximation has been obtained in $[50,86]$, namely, a relation between force and velocity of the form

$$
v=a \omega_{+}(\bar{f}+\kappa a / 2) Q\left(\frac{\omega_{+}^{\prime}(\bar{f}+\kappa a / 2)}{\omega_{+}(\bar{f}+\kappa a / 2)} \kappa a\right)
$$

where the function $Q(x)$ can be approximated by

$$
Q(x) \cong 1 / \sqrt{1+\left(e^{-\gamma} x\right)^{2}}
$$

with $\gamma=0.5772156649 \ldots$ being Euler's constant. The accuracy of this approximation is better than a few per cent for all values of $x$. The relation (45) is valid for arbitrary stiffness $\kappa$, but fast pulling, so that the back-slips are absent. For large $\kappa$, the argument of the $Q$-function becomes large, allowing us to replace $Q(x)$ with $e^{\gamma} / x$. Then, the equation for the mean slip force $\bar{f}_{\text {slip }}=\bar{f}+\kappa a / 2$ becomes almost identical to the one for most probable force, Eq. (40):

$$
v=e^{\gamma} \frac{\omega_{+}^{2}(\bar{f}+\kappa a / 2)}{\kappa \omega_{+}^{\prime}(\bar{f}+\kappa a / 2)} .
$$

The solution is the same as Eq. (42), but with $v$ replaced by $e^{-\gamma} v$ and $f_{*}$ replaced by $\bar{f}+\kappa a / 2$.

\section{F. Force probability distribution}

Because of the "advanced" and "retarded" terms on the right-hand side, an analytical solution of the rate equation in the force domain (31) subject to the normalization condition (30) is a highly non-trivial task. Analytical results can be obtained for the asymptotic cases of small and large stiffness $\kappa$, but for arbitrary pulling velocities. For this, it is convenient 

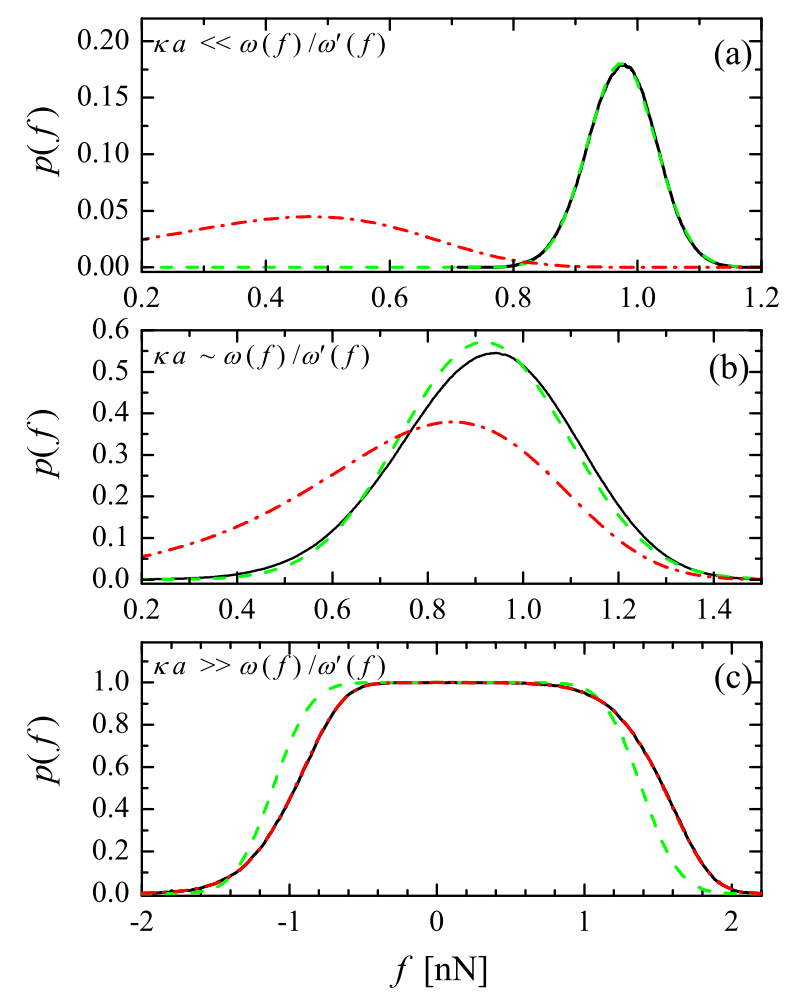

FIG. 4: Force probability distribution $p(f)$ for velocity $v=1 \mu \mathrm{m} / \mathrm{s}$ and other parameters as in Fig. 3. The three sets of data were obtained for different values of the stiffness, namely $\kappa=$ $0.1 \mathrm{~N} / \mathrm{m}(\mathrm{a}), 1 \mathrm{~N} / \mathrm{m}(\mathrm{b})$, and $10 \mathrm{~N} / \mathrm{m}$ (c), corresponding to small, intermediate, and large $\kappa a$. Black solid line: Monte Carlo simulation of the rate equation [82]. Red dash-dotted line: high- $\kappa a$ approximation (48), (50). Green dashed line: low- $\kappa a$ approximation (48), (51). The black and the green curves practically coincide in panel (a). In panel (c), the red and the black curves are almost indistinguishable.

to look for the solution of Eq. (31) in the form that respects the normalization condition (30) from the outset, namely,

$$
p(f)=P(f)-P(f-\kappa a)
$$

such that the new unknown function $P(f)$ monotonically increases between two extreme values $P(-\infty)$ and $P(\infty)$ related by

$$
P(\infty)-P(-\infty)=1
$$


It has been found [82] that, for large $\kappa$, the asymptotic result is

$$
P(f)=\frac{1}{\kappa v} \int_{-\infty}^{f} d f^{\prime} e^{-\frac{1}{\kappa v} \int_{f^{\prime}}^{f} d f^{\prime \prime}\left[\omega_{+}\left(f^{\prime \prime}+\kappa a\right)+\omega_{-}\left(f^{\prime \prime}\right)\right]} \omega_{+}\left(f^{\prime}+\kappa a\right)
$$

and for small $\kappa$, it is

$$
P(f)=\frac{1}{2} \operatorname{erf} \frac{f-\bar{f}+\kappa a / 2}{\sigma}
$$

where the mean force and the force dispersion are given by

$$
v=a \omega_{+}(\bar{f}) \frac{\sinh x}{x} e^{\frac{x}{2}[1-L(x)]}\left(1-e^{-4 \bar{f} x /(\kappa a)}\right), \quad \sigma=\kappa a \sqrt{\frac{1-L(x)}{2 x}},
$$

with $x:=\frac{\kappa a}{2} \frac{d \ln \omega_{+}(\bar{f})}{d f}$ and $L(x):=\operatorname{coth} x-1 / x$ (Langevin function). The high accuracy of these expressions is demonstrated in Fig. 4.

\section{CONCLUDING REMARKS}

The equations used in stochastic modeling, in particular, Langevin equation and rate theory, are not exact laws of nature. Rather, they represent a useful approximation that accounts for the heat-bath effects on a nanoscopic system. In comparison to direct molecular dynamics, they have a number of advantages. Their simplicity often makes them amenable to analytical treatment, whose results can be used to interpret experimental findings. Even when analytical studies of stochastic models are difficult, they still can be easily simulated numerically, with the simulation time being orders of magnitude faster than in the all-atom molecular dynamics approach. Stochastic models can usually be generalized to include additional experimental factors, such as the multidimensional nature of the problem, additional slow degrees of freedom, time-dependent external forcing, etc. Such modifications can be motivated by new experimental results, or introduced with the purpose of designing future experiments that would allow us to discover new phenomena in the nanofriction research area.

\section{ACKNOWLEDGMENTS}

We are grateful to the Deutsche Forschungsgemeinschaft (RE 1344/7-1) and the ESF programs NATRIBO and FANAS (collaborative research project Nanoparma) for financial 
support.

[1] F.P. Bowden and D. Tabor, The Friction and Lubrication of Solids (Oxford Univ. Press, New York, 2008).

[2] M. Urbakh, J. Klafter, D. Gourdon, and J. Israelachvili, Nature 430, 525 (2004).

[3] N. J. Mosey, M. H. Müser, and T. K. Woo, Science 307, 1612 (2005).

[4] J. Phys.: Condens. Matter 20, (35) (2008), special section on friction at the nanoscale.

[5] C.M. Mate, G.M. McClelland, R. Erlandsson, and S. Chiang, Phys. Rev. Lett. 59, 1942 (1987).

[6] G. Binning, C.F. Quate, and Ch. Gerber, Phys. Rev. Lett. 56, 930 (1986).

[7] C.M. Mate, Trobology on the Small Scale (Oxford Univ. Press, New York, 2008).

[8] G.V. Dedkov, Physics Uspekhi 43, 541 (2000).

[9] B.N.J. Persson, Sliding Friction (Springer, Berlin, 2000).

[10] E. Gnecco, R. Bennewitz, T. Gyalog, and E. Meyer, J. Phys.: Condens. Matter 13, R619 (2001).

[11] I. Szlufarska, M. Chandross, and R.W. Carpick, J. Phys. D: Appl. Phys. 41, 123001 (2008).

[12] Y. Dong, A. Vadakkepatt, and A. Martini, Tribol. Lett. 44, 367 (2011).

[13] A. Vanossi, N. Manini, M. Urbakh, S. Zapperi, and E. Tosatti, Rev. Mod. Phys. 85, 529 (2013).

[14] E. Gnecco, R. Bennewitz, T. Gyalog, Ch. Loppacher, M. Bammerlin, E. Meyer, and H.J. Güntherodt, Phys. Rev. Lett. 84, 1172 (2000).

[15] Y. Sang, M. Dubé, and M. Grant, Phys. Rev. Lett. 87, 174301 (2001).

[16] O.K. Dudko, A.E. Filippov, J. Klafter, and M. Urbakh, Chem. Phys. Lett. 352, 499 (2002).

[17] E. Riedo, E. Gnecco, R. Bennewitz, E. Meyer, and H. Brune, Phys. Rev. Lett. 91, 084502 (2003).

[18] S. Sills and R. Overney, Phys. Rev. Lett. 91, 095501 (2003).

[19] M. R. Sorensen, K. W. Jacobsen, and P. Stoltze, Phys. Rev. B 53, 2101 (1996).

[20] A. I. Livshits and A. L. Shluger, Phys. Rev. B 56, 12482 (1997).

[21] G. Gao, R.J. Cannara, R.W. Carpick, and J.A. Harrison, Langmuir 23, 5394 (2007).

[22] A. Martini, Y. Dong, D. Perez, and A. Voter, Tribol. Lett. 36, 63 (2009).

[23] D. Perez, Y. Dong, A. Martini, and A.F. Voter, Phys. Rev. B 81, 245415 (2010). 
[24] W.K. Kim and M.L. Falk, Modelling Simul. Mater. Sci. Eng. 18, 034003 (2010).

[25] W.K. Kim and M.L. Falk, Phys. Rev. B 84, 165422 (2011).

[26] H. Grabert, Projection operator techniques in nonequilibrium statistical mechanics (Springer, Berlin, 1982).

[27] P. Hänggi and H. Thomas, Phys. Rep. 88, 207 (1982).

[28] H. Risken, The Fokker-Planck equation (Springer, Berlin, 1984).

[29] P. Hänggi, P. Talkner, and M. Borkovec, Rev. Mod. Phys. 62, 251 (1990).

[30] P. Reimann, Phys. Rep. 361, 57 (2002).

[31] M. Evstigneev and P. Reimann, Phys. Rev. B 82, 224303 (2010).

[32] L. Prandtl, Zeitschr. f. Angew. Math. u. Mech. 8, 85 (1928).

[33] G.A. Tomlinson, Philos. Mag. 7, 905 (1929).

[34] M. A. Lantz, S. J. O’Shea, M. E. Welland, and K. L. Johnson, Phys. Rev. B 55, 10776 (1997).

[35] M. A. Lantz, S. J. O’Shea, A. C. F. Hoole, and M. E. Welland, Appl. Phys. Lett. 70, 970 (1997).

[36] J. Colchero, A. M. Baró, and O. Marti, Tribology Lett. 2, 327 (1996).

[37] R. W. Carpick, D. F. Ogletree, and M. Salmeron, Appl. Phys. Lett. 70, 1548 (1997).

[38] K. L. Johnson and J. Woodhouse, Tribology Lett. 5, 155 (1998).

[39] R. Bennewitz, T. Gyalog, M. Guggisberg, M. Bammerlin, E. Meyer, and H.-J. Güntherodt, Phys. Rev. B 60, R11301 (1999).

[40] R. Kubo, Rep. Progr. Phys. 29, 255 (1966).

[41] P. Reimann, Chem. Phys. 268, 337 (2001).

[42] P. Reimann and M. Evstigneev, Phys. Rev. Lett. 93, 230802 (2004).

[43] P. Reimann and M. Evstigneev, New Journ. Phys. 7, 25 (2005).

[44] A. Socoliuc, R. Bennewitz, E. Gnecco, and E. Meyer, Phys. Rev. Lett. 92, 134301 (2004).

[45] O.J. Furlong, S.J. Manzi, V.D. Pereyra, V. Bustos, and W.T. Tysoe, Tribol. Lett. 39, 177 (2010).

[46] O.Y. Fajardo and J.J. Mazo, Phys. Rev. B 82, 035435 (2010).

[47] A.E. Filippov, A. Vanossi, and M. Urbakh, Phys. Rev. Lett. 104, 074302 (2010).

[48] O.Y. Fajardo and J.J. Mazo, J. Phys.: Condens. Mat. 23, 355008 (2011).

[49] A.E. Filippov and V.L. Popov, Phys. Rev. E. 75, 027103 (2007).

[50] M. Evstigneev, A. Schirmeisen, L. Jansen, H. Fuchs, and P. Reimann, Phys. Rev. Lett. 97, 
240601 (2006).

[51] Y. Hoshi,T. Kawagishi, and H. Kawakatsu, Jpn. J. Appl. Phys., Part 1 39, 3804 (2000).

[52] R. Roth, Th. Glatzel, P. Steiner, E. Gnecco, A. Baratoff, and E. Meyer, Tribol. Lett. 39, 63 (2010).

[53] M. Reinstädtler, U. Rabe, V. Scherer, U. Harmann, A. Goldade, B. Bhushan, and W. Arnold, Appl. Phys. Lett. 82, 2604 (2003).

[54] S.Yu. Krylov, K.B. Jinesh, H. Valk, M. Dienwiebel, and J.W.M. Frenken, Phys. Rev. E 71, $065101(2005)$.

[55] J. Nakamura, S. Wakunami, and A. Natori, Phys. Rev. B 72, 235415 (2005).

[56] S.N. Medyanik, W.K. Liu, I.-H. Sung, and R.W. Carpick, Phys. Rev. Lett. 97, 136106 (2006).

[57] E. Gnecco, R. Roth, and A. Baratoff, Phys. Rev. B 86, 035443 (2012).

[58] J.S. Helman, W. Baltensperger, and J.A. Hołyst, Phys. Rev. B. 49, 3831 (1994).

[59] W.G. Conley, A. Raman, and C.M. Krousgrill, J. Appl. Phys. 98, 0535519 (2005).

[60] M. Evstigneev and P. Reimann, Phys. Rev. B 73, 113401 (2006).

[61] Z. Tshiprut, S. Zelner, and M. Urbakh, Phys. Rev. Lett. 102, 136102 (2009).

[62] L. Jansen, H. Hölscher, H. Fuchs, and A. Schirmeisen, Phys. Rev. Lett. 104, 256101 (2010).

[63] S.Yu. Krylov, J.A. Dijksman, W.A. van Loo, and J.W.M. Frenken, Phys. Rev. Lett. 97, 166103 (2006).

[64] S.Yu. Krylov and J.W.M. Frenken, New J. Phys. 9, 398 (2007).

[65] S. Yu. Krylov and J.W.M. Frenken, Phys. Rev. B 80, 235435 (2009).

[66] Y. Dong, H. Gao, and A. Martini, EPL 98, 16002 (2012).

[67] Z. Tshiprut, A.E. Filippov, and M. Urbakh, J.Phys.: Condens. Matter. 20, 354002 (2008).

[68] C. Fusco and A. Fasolino, Phys. Rev. B 71, 045413 (2005).

[69] J. Kerssemakers and J.Th.M. De Hosson, Appl. Phys. Lett. 67, 347 (1995).

[70] P. Steiner, R. Roth, E. Gnecco, A. Baratoff, S. Maier, Th. Glatzel, and E. Meyer, Phys. Rev. B 79, 045414 (2009).

[71] E. Gnecco, O.Y. Fajardo, C.M. Pina, and J.J. Mazo, Trib. Lett. 48, 33 (2012).

[72] Z. Tshiprut, A.E. Filippov, and M. Urbakh, Phys. Rev. Lett. 95, 016101 (2005).

[73] A. Socoliuc, E. Gnecco, S. Maier, O. Pfeiffer, A. Baratoff, R. Bennewitz, and E. Meyer, Science 313, 207 (2006).

[74] S. Jeon, T. Thundat, and Y. Braiman, Appl. Phys. Lett. 88, 214102 (2006). 
[75] R. Guerra, A. Vanossi, and M. Urbakh, Phys. Rev. E 78, 036110 (2008).

[76] R. Capozza, A. Vanossi, A. Vezzni, and S. Zapperi, Phys. Rev. Lett. 103, 085502 (2009).

[77] O.Y. Fajardo, R. Roth, E. Meyer, J.J. Mazo, and E. Gnecco, submitted.

[78] R.L. Stratonovich, Radiotekhn. Elektron. (Moscow) 3, 497 (1958). English translation in: P.I. Kuznetsov, R.L. Stratonovich, and V.I. Tikhonov, ed., Non-linear Transformations of Stochastic Processes (Pergamon, Oxford, 1965).

[79] G.I. Bell, Science 200, 618 (1978).

[80] M. Muser, Phys. Rev. B 84, 125419 (2011).

[81] K.B. Jinesh, S.Yu. Krylov, H. Valk, M. Dienwiebel, and J.W.M. Frenken, Phys. Rev. B 78, 155440 (2008).

[82] M. Evstigneev and P. Reiman, Phys. Rev. B 87, 205441 (2013).

[83] M. Evstigneev, Phys. Rev. E 78, 011118 (2008).

[84] M. Evstigneev, A. Schirmeisen, L. Jansen, H. Fuchs, and P. Reimann, J. Phys.: Condens. Matt. 20, 354001 (2008).

[85] O.J. Furlong, S.J. Manzi, V.D. Pereyra, V. Bustos, and W.T. Tysoe, Phys. Rev. B 80, 153408 (2009).

[86] M. Evstigneev and P. Reimann, Phys. Rev. E 71, 056119 (2005).

[87] S. Maier, Y. Sang, T. Filleter, M. Grant, R. Bennewitz, E. Gnecco, and E. Meyer, Phys. Rev. B 72, 245418 (2005).

[88] A. Schirmeisen, L. Jansen, and H. Fuchs, Phys. Rev. B 71, 245403 (2005). 